\title{
An integrated digital/clinical approach to smoking cessation in lung cancer screening: study protocol for a randomized controlled trial
}

Amanda L. Graham ${ }^{1,2^{*}}$, Michael V. Burke², Megan A. Jacobs', Sarah Cha', Ivana T. Croghan ${ }^{3,4}$, Darrell R. Schroeder ${ }^{5}$, James P. Moriarty ${ }^{6}$, Bijan J. Borah, Donna F. Rasmussen ${ }^{3}$, M. Jody Brookover ${ }^{1}$, Dale B. Suesse ${ }^{8}$,

David E. Midthun ${ }^{9}$ and J. Taylor Hays $s^{3,10}$

\begin{abstract}
Background: Delivering effective tobacco dependence treatment that is feasible within lung cancer screening (LCS) programs is crucial for realizing the health benefits and cost savings of screening. Large-scale trials and systematic reviews have demonstrated that digital cessation interventions (i.e. web-based and text message) are effective, sustainable over the long-term, scalable, and cost-efficient. Use of digital technologies is commonplace among older adults, making this a feasible approach within LCS programs. Use of cessation treatment has been improved with models that proactively connect smokers to treatment rather than passive referrals. Proactive referral to cessation treatment has been advanced through healthcare systems changes such as modifying the electronic health record to automatically link smokers to treatment.

Methods: This study evaluates the impact of a proactive enrollment strategy that links LCS-eligible smokers with an evidence-based intervention comprised of a web-based (WEB) program and integrated text messaging (TXT) in a three-arm randomized trial with repeated measures at one, three, six, and 12 months post randomization. The primary outcome is biochemically confirmed abstinence at 12 months post randomization. We will randomize 1650 smokers who present for a clinical LCS to: (1) a usual care control condition (UC) which consists of Ask-Advise-Refer; (2) a digital (WEB + TXT) cessation intervention; or (3) a digital cessation intervention combined with tobacco treatment specialist (TTS) counseling (WEB + TXT + TTS).

(Continued on next page)
\end{abstract}

\footnotetext{
* Correspondence: agraham@truthinitiative.org

1 Schroeder Institute for Tobacco Research and Policy Studies at Truth

Initiative, 900 G Street NW, 4th Floor, Washington, DC 20001, USA

${ }^{2}$ Department of Oncology, Georgetown University Medical Center/Cancer

Prevention and Control Program, Lombardi Comprehensive Cancer Center,

Washington, DC, USA

Full list of author information is available at the end of the article
} 
(Continued from previous page)

Discussion: The scalability and sustainability of a digital intervention may represent the most cost-effective and feasible approach for LCS programs to proactively engage large numbers of smokers in effective cessation treatment. We will also evaluate the impact and cost-effectiveness of adding proven clinical intervention provided by a TTS. We expect that a combined digital/clinical intervention will yield higher quit rates than digital alone, but that it may not be as cost-effective or feasible for LCS programs to implement. This study is innovative in its use of interoperable, digital technologies to deliver a sustainable, scalable, high-impact cessation intervention and to facilitate its integration within clinical practice. It will add to the growing knowledge base about the overall effectiveness of digital interventions and their role in the healthcare delivery system.

Trial registration: ClinicalTrials.gov, NCT03084835. Registered on 9 March 2017.

Keywords: Cancer screening, Lung cancer, Smoking cessation, Internet, Text messages, Tobacco use cessation, Counseling

\section{Background}

Lung cancer screening (LCS) provides a unique opportunity to deliver tobacco dependence treatment on a population-wide basis. More than 4 million current smokers age $55+$ years are likely to be eligible for LCS $[1,2]$. LCS saves lives through early identification of lung cancer. However, given that most lung screens are negative [2] and that smoking causes 11 other types of cancer as well as cardiovascular disease and other lung disease [3], the health benefits of providing effective tobacco dependence treatment for LCS patients are far-reaching. Smokers seeking LCS report interest in quitting and receiving cessation services [4-8] and simulation models predict that tobacco dependence treatment can improve the cost-effectiveness of LCS by $20-50 \%$ [9, 10]. Delivering tobacco dependence treatment that is feasible within a LCS program is crucial for maximizing the health benefits of screening [11, 12].

The Centers for Medicare and Medicaid Services (CMS) requires that LCS include a shared decisionmaking visit with smokers that includes "counseling on the importance of smoking cessation and furnishing of information about tobacco cessation interventions" [13]. This approach is analogous to the Ask-Advise-Refer (AAR) model of tobacco dependence treatment [6] in which healthcare providers ask about smoking status, advise a smoker to quit, and refer to readily available cessation treatment resources. A recent study by Ostroff et al. [14] reported that the majority of LCS programs "always" ask patients about smoking status (98.9\%) and advise current smokers to quit (91.4\%) but fewer reported delivery of cessation counseling (57\%) or referral to a quit line $(60.2 \%)$. Low rates of treatment referral in LCS programs are consistent with data from other healthcare settings [15-17]. Additionally, most smokers passively referred to treatment fail to follow through [15-18]. As noted by Fucito et al. [19], "patient motivation as a requirement for treatment (i.e. an opt-in model) means that most smokers will not receive smoking-cessation assistance" (page 9). Other treatment models are needed to address smoking cessation in the LCS setting.

Use of cessation treatment has been improved with models that proactively connect smokers to treatment resources (i.e. an opt-out model) rather than simply advising smokers to quit and providing referral information [20, 21]. Historically done through e-mail or fax, clinic staff sends a smoker's contact information to a cessation treatment program - most often a quit line followed by attempts by the cessation program to proactively reach the smoker. More recently, treatment uptake and engagement have been advanced through healthcare systems changes such as modifying the electronic health record (EHR) to automatically link smokers to tobacco dependence treatment [22-24]. Referred to as Ask-Advise-Connect (AAC), the healthcare provider asks about smoking status, advises a smoker to quit, and then immediately connects the smoker with cessation treatment by sending their contact information directly to a cessation program via an automated process through the EHR followed by proactive strategies by the program to engage the smoker in treatment. AAC is designed to address both clinic-level and patient-level barriers to treatment enrollment and has shown great promise. Compared to AAR, AAC yielded significant increases in quit line enrollment among smokers in a private healthcare system [25] and a safety net healthcare system [26].

The AAC approach combined with digital cessation interventions represents an exciting and novel approach to engaging smokers in treatment. The scalability and sustainability of a digital intervention may represent the most cost-effective and feasible approach for LCS programs to proactively engage large numbers of smokers 
in cessation treatment. Web-based (WEB) cessation programs are well-suited to deliver evidence-based cessation treatment. Problem-solving and skills-training information can be provided in interactive exercises; social support from peers and experts can be provided in an online community; and information and guidance about pharmacotherapy use can be provided. Unlike other treatment modalities, WEB interventions can be accessed 24/7 - particularly at relapse sensitive times and used for as long as a smoker desires. The need for more extended and intensive cessation treatment strategies within the LCS setting was noted in a recent systematic review [27]. Large-scale trials of Internet interventions have reported quit rates of $18-20 \%$ at one year $[28,29]$ and several meta-analyses provide evidence of effectiveness, especially for programs that are interactive and tailored [30-33]. WEB cessation programs are cost-effective [34, 35] and scalable [35-38], able to accommodate thousands of additional users at little incremental cost. Evidence in support of AAC with digital interventions is beginning to emerge. Ray et al. [39] conducted a randomized trial in dental practices of electronic vs paper referral to an interactive, tailored cessation website. The proportion of smokers that registered on the website was fourfold higher among clinics using e-referral compared to clinics using paper referral. Abstinence rates were also significantly higher among ereferral clinics. The cost per registered smoker was in the range of $\$ 67-\$ 87$ [40], making this an extremely cost-efficient approach to connect smokers with evidence-based treatment. As a comparison, provider referrals using brochures yielded a cost of $\$ 597$ per smoker recruited to a WEB intervention [41].

Text messaging (TXT) is also a proven cessation strategy [42]. Automated TXT programs can be designed to conform to established guidelines for smoking cessation [43] and can mirror elements of in-person counseling such as goal-setting and feedback, social support, and a personalized quit plan [44, 45]. TXT programs can be delivered long term $[46,47]$ and can increase the contact time and intensity of adjunct treatments [43]. The efficacy [48] and cost-effectiveness [49] of TXT programs for cessation are well-documented.

Current data and well-documented trends within healthcare substantiate the relevance of technologybased cessation interventions for older adults. Technology use is high and growing among older adults who are candidates for LCS (i.e. those aged 55 years and older [13]). Internet use and cell phone adoption among older adults continue to increase steadily: as of November 2016, 87\% of those aged 50-64 years were Internet users, and 6/10 seniors (aged 65+ years) reported they go online [50]. The Internet is a trusted source of health information among older adults [51]. Approximately
$74 \%$ of older adults (aged $60+$ years) and $88 \%$ of Baby Boomers (aged 51-59 years) use a cellular device and an increasing number now have mobile Internet access [52]. Technology use among older adults is expected to become even more ubiquitous as the 78 million techsavvy Baby Boomers grow older [53] and as national trends in healthcare and advances in health information technology continue to drive technology use among older adults [54-56].

Given that many patients seen in LCS programs will be older, long-term, heavy smokers with varying levels of interest in quitting [57], more intensive clinical intervention provided by a tobacco treatment specialist (TTS) may be necessary. TTS counseling has been integrated into a range of healthcare settings [58-60] and yielded quit rates in the range of $26.5-34 \%$ [43, 59-64]. A TTS working within a LCS program can provide more efficacious treatment than non-specialists or brief physician counseling [62, 65, 66]. Preliminary evidence suggests that implementing a TTS intervention before LCS may be more effective in promoting abstinence than interventions delivered after LCS. A pilot study by Ferketich et al. [67] reported six-month carbon monoxide (CO)confirmed abstinence rates of $22 \%$ among smokers that received TTS counseling and pharmacotherapy before LCS compared with $11.1 \%$ among smokers that received the intervention after LCS. Treatment approaches that combine TTS counseling and digital interventions improve cessation outcomes and can be adopted in different types of healthcare systems [68]. Several large-scale studies have found that TTS counseling combined with a WEB cessation intervention yields significantly higher quit rates than a WEB intervention alone [29, 69]. To date, we are not aware of any studies that have evaluated proactive enrollment into a combined digital and clinical cessation intervention in LCS programs.

Building on the evidence in support of AAC models, this hybrid effectiveness-implementation trial will evaluate the impact of a proactive enrollment strategy that links smokers seeking LCS with a digital cessation intervention comprising a WEB program and integrated TXT alone and in combination with TTS counseling. Our trial is guided by the RE-AIM framework [70] which provides a systematic way to evaluate the impact of the implementation of public health interventions on five criteria: reach; efficacy/effectiveness; adoption; implementation; and maintenance. This study will focus on reach, effectiveness, and adoption. The primary outcome is biochemically confirmed smoking abstinence at 12 months. The specific aims are as follows: Aim 1 (Effectiveness): to evaluate the comparative effectiveness of WEB + TXT and WEB + TXT + TTS vs a usual care (UC) AAR control group. Hypothesis 1a: $\mathrm{WEB}+\mathrm{TXT}+\mathrm{TTS}$ 
yields higher quit rates than WEB + TXT and UC. $H y$ pothesis 1b: WEB + TXT yields higher quit rates than UC; Aim 2 (Implementation): to examine whether proactive enrollment increases treatment use and whether treatment use mediates the relationship between treatment assignment and smoking outcome. Hypothesis 2a: Proactive enrollment in WEB + TXT will yield greater levels of treatment use than passive referral to the same interventions in UC. Hypothesis $2 b$ : Increases in treatment use will mediate the relationship between treatment group and outcome; Aim 3 (Reach and Adoption): to examine the representativeness of the study sample relative to all smokers screened for LCS eligibility (reach) and the potential for intervention implementation (adoption) among LCS clinics.

Our trial is structured as a practical clinical trial [71] to answer key questions about implementation and effectiveness of direct relevance to decision-makers. The evaluation of interoperable, digital technologies to deliver a sustainable, scalable cessation intervention and to facilitate its integration within clinical practice will add to the growing knowledge base about the overall effectiveness of digital interventions and their role in the healthcare delivery system.

\section{Methods/design}

This randomized clinical trial follows the SPIRIT guidelines (see Additional file 1). The study schedule of enrollment, interventions, and assessments is presented in Fig. 1.

\section{Study setting}

This multisite trial will include a mix of healthcare sites serving patients from varied and diverse communities across four states: four sites from the Mayo Clinic Health System; two Mayo Clinic campuses in urban areas; and a destination medical center in Rochester, $\mathrm{MN}$, that serves patients from all 50 states. These sites include a mix of hospital and community centers that are located throughout the US (upper Midwest, Southwest, Southeast) and facilities that are large and small, urban and rural. This variety in study sites will ensure generalizability to other healthcare systems and LCS programs in moderate to large healthcare facilities.

Across study sites, all aspects of LCS and clinical trial management will be facilitated by the Big One Stop Shop (BOSS) Registry, an in-house, Mayo-owned solution that serves as a surrogate for Mayo Clinic's planned system-wide, single-instance EHR. The BOSS Registry is a WEB system that is highly scalable and used by various groups in research and clinical practice. It will be

\begin{tabular}{|c|c|c|c|c|c|c|c|}
\hline \multirow[b]{3}{*}{ TIMEPOINT } & \multicolumn{7}{|c|}{ Study Period } \\
\hline & \multirow{2}{*}{$\begin{array}{c}\text { Enrollment } \\
0\end{array}$} & \multirow{2}{*}{$\begin{array}{c}\text { Allocation } \\
0\end{array}$} & \multicolumn{4}{|c|}{ Post-allocation } & \multirow{2}{*}{$\begin{array}{c}\text { Close-out } \\
12 \mathrm{mo}\end{array}$} \\
\hline & & & $1 \mathrm{mo}$ & $3 \mathrm{mo}$ & $6 \mathrm{mo}$ & $12 \mathrm{mo}$ & \\
\hline \multirow{5}{*}{$\begin{array}{l}\text { ENROLLMENT: } \\
\text { Shared decision making } \\
\text { Eligibility screen } \\
\text { Informed consent } \\
\text { Allocation }\end{array}$} & & & & & & & \\
\hline & $x$ & & & & & & \\
\hline & $x$ & & & & & & \\
\hline & $x$ & & & & & & \\
\hline & & $x$ & & & & & \\
\hline \multirow{4}{*}{$\begin{array}{l}\text { INTERVENTIONS: } \\
\text { Usual Care } \\
\text { WEB+TXT } \\
\text { WEB+TXT+TTS }\end{array}$} & & & & & & & \\
\hline & & $x$ & & & & & \\
\hline & & $x$ & 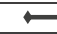 & & & $\Rightarrow$ & \\
\hline & & $x$ & 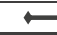 & & & $\Rightarrow$ & \\
\hline \multirow[b]{2}{*}{$\begin{array}{l}\text { ASSESSMENTS: } \\
\text { Baseline: } \\
\text { - Demographics } \\
\text { - Perceived vulnerability \& worry } \\
\text { about lung cancer } \\
\text { - Current smoking \& smoking } \\
\text { - Mistory } \\
\text { - Quitivation to quit smoking } \\
\text { - Nicotine dependence } \\
\text { - Quality of life questionnaire }\end{array}$} & & & & & & & \\
\hline & $x$ & & & & & & \\
\hline \multirow{2}{*}{$\begin{array}{l}\text { Primary Outcome: CO-verified } \\
\text { smoking abstinence } \\
\\
\text { Secondary Outcomes: } \\
\text { - Motivation to quit smoking } \\
\text { - \# of quit attempts } \\
\text { - 30-day and continuous } \\
\text { abstinence } \\
\text { - Intervention satisfaction } \\
\text { - Quality of life questionnaire }\end{array}$} & & & & & & & $x$ \\
\hline & & & $\mathrm{x}$ & $x$ & $x$ & $x$ & $x$ \\
\hline $\begin{array}{l}\text { Medical Outcomes: } \\
\text { - Lung cancer screening results }\end{array}$ & & & & & & & $\mathrm{x}$ \\
\hline
\end{tabular}

Fig. 1 SPIRIT figure 
adapted to store baseline assessment data specific to this trial, house the randomization scheme, and notify the site Study Coordinator when potential participants schedule a shared decision-making visit. The BOSS Registry will also be modified to allow for secure exchange of data with the BecomeAnEX system immediately upon randomization and throughout the study.

\section{Participants}

We will recruit 1650 current smokers who complete a LCS shared decision-making visit. Patients will be eligible regardless of whether it is their first shared decision-making visit or not. Study eligibility criteria are: (1) current smoking (every day/some days in the past 30 days); (2) at least weekly use of the Internet; (3) cell phone ownership with a TXT plan; and (4) willingness to receive study text messages. Exclusion criteria are: current use of cessation medication; participation in tobacco cessation treatment; or failure to sign informed consent.

\section{LCS eligibility screening and shared decision-making} Patients can self-refer for LCS or be referred by a medical provider. Interest in quitting or in participating in a smoking cessation program are not prerequisites for self-referral or provider-referral. Patients will complete a telephone screening during which demographic information, smoking history, current smoking status, and other data for risk calculation are collected. Eligibility criteria for LCS (per US Preventive Services Task Force) are: (1) age 55-80 years; (2) 30 + -pack/year history of smoking; (3) current smoker or one who has quit smoking within the last 15 years; and (4) asymptomatic (no signs or symptoms of lung cancer) [72]. The Mayo Clinic LCS program also recommends screening to those who are at high risk with a $\mathrm{PLCO}_{\mathrm{m} 2012}$ risk calculation of $\geq 1.34 \%$ over the next six years, but who do not meet USPSTF criteria [73]. If the patient is eligible for LCS, a face-toface shared decision-making session is scheduled during which the risks and benefits of LCS - and the benefits of quitting smoking - are described and patients are helped to make an independent decision about whether to proceed with LCS. Medicare covers payment for LDCT screening for those aged 65-74 years with a 30 + -pack/year history; patients who are no longer smoking must have quit within 15 years. Programs of the Affordable Care Act cover those who meet USPSTF criteria. Private insurance or out-of-pocket payment is required for those who are at high-risk for lung cancer but do not meet CMS or USPSTF criteria.

\section{Study recruitment and informed consent}

The site Study Coordinator will be notified by the BOSS Registry when an eligible, current smoker is scheduled for shared decision-making. At the end of shared decision-making, current smokers who decide to proceed with LCS will be presented with study information. Interested smokers will complete study eligibility screening, sign informed consent, and complete a computerized baseline assessment. Smoking cessation treatment will be initiated immediately following the baseline assessment per the randomization assignment.

\section{Randomization}

Randomization will be automated by computer algorithm and stratified by study site.

\section{Overview of smoking cessation interventions}

All study participants will be given an educational booklet about pharmacotherapy called Medications to Help You Stop Using Tobacco [74]. Developed at Mayo Clinic, this booklet provides the rationale and instructions for using medications and describes the mechanisms by which medications work. It is not possible to blind participants to intervention assignment given the nature of the interventions. However, the two experimental arms are standardized and/or delivered according to a protocol.

\section{Usual care (UC) control arm}

This arm represents tobacco dependence treatment within LCS programs as mandated by CMS. The site Study Coordinator will conduct brief cessation counseling ( $<3 \mathrm{~min})$. Consistent with the AAR approach [6], this discussion will involve asking about tobacco use, advising the participant about the importance of quitting smoking, and referring the participant to cessation treatment. Referral to treatment in this arm will be passive, consisting of printed information about the digital intervention (BecomeAnEX website and TXT program, details below) and Mayo Clinic TTS services. To facilitate detailed tracking of digital treatment use among participants that follow through with either of these referrals, we will provide a custom URL for the BecomeAnEX website and a study-specific text message enrollment number. This will enable us to identify Arm 1 patients in the BecomeAnEX database and evaluate digital treatment use in a comparable manner across all three treatment arms.

\section{WEB + TXT intervention}

The site Study Coordinator will conduct brief cessation counseling ( $<3 \mathrm{~min})$ and then proactively enroll participants in the digital intervention using a study-specific enrollment page on BecomeAnEX. After locating the participant in the BecomeAnEX database using a unique study identifier, the Study Coordinator will assist the participant in completing registration for the WEB and 
TXT program and provide a brief orientation about both interventions.

The BecomeAnEX website is Truth Initiative's smoking cessation program that was launched in 2008 [75, 76]. The program was designed around national guidelines for tobacco dependence treatment [43] and principles of social cognitive theory [77]. A national mass media campaign [75] and ongoing online advertising have resulted in over 800,000 registered users since its inception. BecomeAnEX educates smokers about the behavioral and pharmacologic aspects of nicotine addiction and provides tools to enhance self-efficacy for quitting. It guides and supports smokers through a series of interactive components that assist users in selecting a quit date, identifying their smoking triggers, developing coping skills, and selecting a cessation medication. The site also includes a large social network comprising thousands of current and former smokers who interact via several asynchronous communication channels. All user actions are date- and time-stamped and stored in a relational database. BecomeAnEX was extensively pilot tested during development [75] and designed around website usability best practices, making it appropriate for use by older adults [78-81].

The BecomeAnEX TXT program is a fully automated system that has been integrated into the website. The TXT program is also based on social cognitive theory [77]. Messages are tailored around a user-entered quit date and provide general quitting advice, positive reinforcement, motivation, reminders to avoid smoking cues and triggers, and information about nicotine and withdrawal. Keywords (e.g. "CRAVE," "MORE") generate on-demand support and information and "STOP" terminates the program at any time. The program delivers three weeks of oncedaily cessation text messages regardless of whether a user sets a quit date. When a user sets a quit date, they receive two weeks of twice daily messages prequit date and six weeks of 1-3 messages/day post quit date, with periodic messages at cessation milestones (e.g. six months post quit date).

Both the WEB and TXT programs will be customized for delivery to LCS patients. The user will see study information on their individual home page to make clear they have arrived at the correct website. LCS-specific content and videos to reinforce the importance of cessation regardless of LCS results will be added to the website. For the TXT program, we will extend the duration to make it a 12-month intervention to prompt re-engagement with the WEB components and to remind patients of their one-year LCS appointment. Participants will receive four messages per month beyond the standard program described above.

\section{$W E B+T X T+T T S$ intervention}

Initiation of the WEB + TXT intervention in this arm is identical to the approach described above. Unique to this treatment arm is a required session with a TTS before LCS. During this session, the TTS will conduct an assessment, formulate a treatment plan, and provide counseling, education, and arrange for follow-up. TTS counselors will be able to view real-time utilization data from BecomeAnEX through the BOSS Registry, already part of their routine workflow. They will know which elements of the website the participants have used/not used and will be able to incorporate this information into the counseling session. The TTS will utilize motivational interviewing skills and strategies to create momentum for quitting and actively engage the patient. TTS counseling incorporates information gathered from an assessment to collaboratively develop an individually tailored treatment plan that consists of cognitive and behavioral strategies and pharmacological treatment. An interactive patient treatment guide will be used to standardize the educational and counseling content. At the conclusion of this initial session, the TTS will schedule four additional sessions.

\section{Lung cancer screening}

Low dose computed tomography (LDCT) LCS will be conducted in the Mayo Lung Screening Program. All sites are designated as American College of Radiology (ACR) LCS centers. The parameters of the screening at each participating site are done in accordance with ACR and CMS requirements [82]. The screening is done with the patient lying in the scanner with arms overhead and accomplished in a single breath-hold in $<15 \mathrm{~s}$. The CT is read by a thoracic radiologist who has received ACR accreditation and read using the Lung-RADS (lung imaging reporting and data system) standardized lung nodule reporting scheme as required by ACR [83]. The program collects and submits data to the ACR registry for each LDCT LCS performed. Every patient receives a letter with recommendations and their scan report, which are also sent to their designated practitioner. The next annual screening $\mathrm{CT}$ is recommended for patients with a normal scan or those with one or more nodules < $6 \mathrm{~mm}$; interval follow-up is provided for nodules $<$ $10 \mathrm{~mm}$. Patients with nodule(s) $\geq 10 \mathrm{~mm}$ or adenopathy are referred to a pulmonologist associated with the Mayo Lung Screening Program and pursued in accordance with published guidelines using a multidisciplinary approach.

\section{Assessments}

Assessments will occur at baseline and one, three, six, and 12 months post randomization. Follow-ups will be conducted by telephone by research staff who are not 
blind to treatment assignment but who do not have any role in intervention delivery. We will pilot test measures with 8-10 LCS patients (not participants) to ensure wording and administration are appropriate for older adults [84]. We expect at least $80 \%$ follow-up at 12 months based on previous research by our team [29, $85,86]$ and retention of $>90 \%$ in previous Mayo Clinic LCS studies [87]. To maximize follow-up, we will: (1) provide clear information about the study at the outset, including expectations for follow-up data collection; (2) reimburse participants with graduated incentives for all follow-up data collection, including a separate incentive for CO testing at 12 months; and (3) emphasize the importance of survey completion regardless of smoking status. If follow-up rates are lower than expected, we will consider implementing additional modes of follow-up (e.g. online survey) used successfully by our team at minimal cost.

\section{Baseline variables}

To characterize the sample and examine moderators of treatment effectiveness, the baseline assessment will assess: demographics (age, sex, ethnicity, race, marital status, education, number of household tobacco users); perceived vulnerability and worry about lung cancer (personal risk of developing cancer, their likelihood compared to other smokers, and their level of worry about getting lung cancer); current smoking and smoking history (smoking frequency and rate, lifetime and past 30day use of other tobacco products and e-cigarettes); motivation to quit smoking will be measured with the Readiness Ladder $[88,89]$ and an item regarding confidence in quitting; quitting history, including past year quit methods; nicotine dependence will be measured with the Fagerström Test for Nicotine Dependence [90].

The following metrics will be extracted from the BOSS Registry for all patients that are determined eligible for LCS: age; education; race/ethnicity; body mass index; referral source; and smoking status. We will compare patients eligible for LCS to study participants on these characteristics to evaluate representativeness of the study sample.

\section{Process variables}

Treatment utilization will be assessed via self-report at each follow-up and automated tracking metrics extracted from BecomeAnEX and the BOSS Registry. At each follow-up, participants will be asked about their use of any cessation methods. The following metrics will be extracted from BecomeAnEX: total time spent logged into the website; number of return visits; use of the static content and videos; use of interactive components/tools; number of replies to interactive text messages; unsubscribe rates; modal day of unsubscribe; number of days enrolled in TXT; and number of text messages received. The following metrics will be extracted from BOSS regarding completion of TTS counseling: number and duration of completed TTS counseling sessions; tobacco use status; confidence and importance for quitting or maintaining abstinence; medication use; cravings/urges and management strategies.

TTS intervention fidelity will be monitored throughout the study via review of digital audio recordings. Audio review will be conducted using a fidelity measure based on the Mayo Clinic treatment model including items adapted from the Motivational Interviewing Treatment Integrity Code [60].

\section{Primary outcome}

The primary outcome is biochemically confirmed (carbon monoxide [CO]) abstinence at 12 months post randomization. All participants who self-report sevenday abstinence at the 12-month follow-up will be invited to provide a breath sample for $\mathrm{CO}$ testing. Expired alveolar $\mathrm{CO}$ concentrations of $\leq 10 \mathrm{ppm}$ will be considered verification of abstinence [91]. Participants who decline testing will be considered smokers.

\section{Secondary outcomes}

Other smoking-related outcomes will include change in motivation to quit, number of quit attempts, and 30-day and continuous abstinence measured at each follow-up [92]. Intervention satisfaction in all three arms will be measured with an adapted version of the Client Satisfaction Questionnaire [93]. We will assess overall satisfaction and perceived helpfulness, whether the intervention met their expectations $(1=$ not at all, $5=$ very much $)$, and whether they would recommend it to a friend (yes/ no). We will include items that address usability of the WEB and TXT interventions by modifying measures from the System Usability Scale (all measures $1=$ strongly disagree, 5 = strongly agree): whether they found them unnecessarily complex; whether they found them easy to use; whether they felt they would need the support of a technical person to be able to use them; whether they felt very confident using them; whether there was too much inconsistency in them; and whether they would imagine that most people would learn to use them very quickly [94]. Quality of life will be measured at baseline and 12-month follow-up with the Smoking Cessation Quality of Life Questionnaire $[95,96]$ and will be assessed with a single item 10-point rating scale at one-, three-, and six-month follow-ups.

\section{Medical outcomes}

BOSS provides the ability to capture radiology results of LDCT of patients with lung nodules such as Lung- 
RADS, follow-up procedures, diagnosis of lung cancer, vital status, and other concurrent diagnoses. Follow-up LCS status will be determined using data from the BOSS Registry at one year (all participants) and two years ( 80\% participants).

\section{Data monitoring}

At the start of the study, a Data Safety Monitoring Committee will be established, comprising the Principal Investigators, Data Analyst, Biostatistician, Technical Lead, and Project Manager. Every two weeks the committee will review participant accrual rate, participant drop-out and the reasons for drop-out, target enrollment status, major and minor problems related to treatment assignment, and serious adverse event reports.

This is a minimal risk study for which no Data Safety Monitoring Board is needed. Standard clinical trial operating procedures for crisis management will be in place throughout the trial. Study staff will monitor and report serious adverse events.

\section{Power calculation}

Positive (i.e. abnormal) LCS findings increase the odds of abstinence [6, 97-99]. Therefore, to be conservative in our sample size calculations, efficacy estimates for UC are based on data from patients in previous trials with negative screening results. Among patients with negative findings in the National Lung Screening Trial, one-year abstinence rates are in the range of 9.7\% [100] to $12.6 \%$ [97]. In the Danish Lung Cancer Screening Trial [98], patients received $<5 \mathrm{~min}$ of counseling from a nurse and 30-day abstinence at one-year follow-up was $11.4 \%$ among patients with no significant LCS findings. Cox et al. [101] reported seven-day abstinence at one year of $13 \%$. Brief counseling for smoking cessation $(<3 \mathrm{~min})$ yields six-month abstinence rates of $13.4 \%$ [43]. Therefore, we conservatively estimate that biochemically verified abstinence at 12 months for UC will be $10 \%$.

Efficacy estimates for WEB + TXT are based on several converging lines of evidence: (1) two meta-analyses have reported statistically significant effects of interactive and individually tailored interventions compared to usual care or written self-help (relative risk $[R R]=1.48,95 \%$ confidence interval $[\mathrm{CI}]=1.11-2.78[30] ; \mathrm{RR}=2.10,95 \%$ $\mathrm{CI}=1.25-3.52$ [33]; (2) a meta-analysis by Webb et al. [102] found that the use of TXT in Internet interventions had large effects on behavior change $(\mathrm{d}=0.81, \mathrm{k}=$ 4); (3) combined Internet and TXT interventions yielded six-month sustained abstinence of $17.1 \%$ in Borland et al. [103] and 12-month repeated point prevalence abstinence of $20 \%$ in Brendryen et al. [46]. Therefore, we conservatively estimate that biochemically verified abstinence at 12 months for WEB + TXT will be $16 \%$.
Efficacy estimates for WEB + TXT + TTS are also based on several lines of evidence: (1) abstinence reported by Fiore et al. [43] for treatment duration of 3190 min (comparable to the TTS visit that will occur before LCS) was $26.5 \%$ (95\% CI =21.5-31.4); (2) among more than 6000 patients who received TTS counseling in the NDC outpatient clinic, seven-day point prevalence abstinence at six months under intention-to-treat (ITT) analysis was $28.1 \%(95 \% \mathrm{CI}=27.7-30.1)$ [60]; (3) in a pilot study of LCS patients who completed a TTS visit before LCS, seven-day abstinence at six months (ITT) was 34\% [64]; (4) previous studies of combined Internet and TTS counseling interventions have yielded quit rates (30-day point prevalence abstinence under ITT) of $21.5 \%$ [29] to $53 \%$ [69] with a strong dose-response relationship for the number of TTS sessions. Therefore, we conservatively estimate that biochemically verified abstinence at 12 months for WEB + TXT + TTS will be $24 \%$.

For pairwise treatment comparisons, we will use the Holm-Š́dák approach to account for multiple comparisons [104]. The three pairwise comparisons will be performed with two-tailed $p$ values obtained which are not adjusted for multiple comparisons. These $p$ values will be ranked from smallest to largest. If the smallest $p$ value is $>0.0167$ we will conclude that none of the comparisons is statistically significant. If the smallest $p$ value is $\leq 0.0167$ this comparison will be considered statistically significant and the second smallest $p$ value will be evaluated. If the second smallest $p$ value is $>0.025$, we will conclude that the final two comparisons are not statistically significant. If the second smallest $p$ value is $\leq$ 0.025 , this comparison will be considered statistically significant and the final $p$ value will be evaluated using $p \leq 0.05$ to denote statistical significance. For our hypothesized abstinence rates, a total sample-size of 1650 (550/group) will provide statistical power $\geq 99 \%$ for the overall comparison across treatment groups and $>85 \%$ for each of the three pairwise treatment comparisons.

\section{Statistical analyses}

Unless otherwise specified, analyses use an ITT approach that analyzes individuals by treatment assignment. We will use descriptive statistics to summarize demographic data, tobacco use history, and other baseline characteristics. Summaries will be generated for the entire sample and by treatment arm. Given the randomized design, we will not assess for significant baseline treatment differences. With the exception of the stratification variable, no covariate adjustment will be included in the primary analysis. For all exploratory analyses, two-tailed $p$ values will be reported with no adjustment for multiple comparisons. In all cases, findings will be reported using point estimates and corresponding 95\% CIs. Model 
assumptions for each analysis will be validated and transformations or non-parametric methods will be utilized as appropriate.

\section{Aim 1 (Effectiveness) analyses}

The primary outcome of biochemically confirmed pointprevalence abstinence at 12 months post randomization will be analyzed using ITT. The primary analysis of the smoking outcomes will be performed using logistic regression. Separate analyses will be performed for each abstinence endpoint. For the logistic regression analyses, the dependent variable will be smoking abstinence and the independent variable will be treatment (WEB + TXT + TTS vs WEB + TXT vs UC). Study site will be included as a covariate in the primary analyses. We hypothesize that WEB + TXT + TTS will yield higher quit rates than $\mathrm{WEB}+\mathrm{TXT}$ and UC and that WEB + TXT will yield higher quit rates than UC. To test these hypotheses, all pairwise comparisons of the three treatment groups will be performed. For the primary analysis of smoking abstinence outcomes, any individual who misses a visit will be classified as smoking for that assessment. This approach is used because the data are assumed to be missing not at random (MNAR). However, this approach is sensitive to differential attrition across treatment groups and tends to overestimate the precision of the estimate of the treatment effect. Therefore, to supplement primary analysis we will perform sensitivity analysis using multiple-imputation based on pattern-mixture models under the MNAR assumption (SAS/STAT version 13.2, PROC MI and PROC MIANALYZE). Multiple imputations of missing abstinence outcomes will be imputed using the logistic regression method, with the outcome adjusted by modifying the log odds ratios (OR) [105, 106]. Since the OR relating missingness to smoking is not known, we will conduct a sensitivity analysis to assess its impact on the estimate and significance of the treatment effects.

We will examine potential moderators (e.g. screening result, gender, age) by analyzing interactions between treatment and selected variables. We will examine treatment/moderator interaction term on outcomes after entering main effects. We will also explore moderators of the relationship between treatment assignment and treatment utilization.

\section{Aim 2 (Implementation) analyses}

First, we will examine treatment uptake and engagement via self-reported treatment use (behavioral or pharmacologic) and automated tracking data from the BecomeAnEX WEB and TXT program at each follow-up interval. We will test whether proactive enrollment in treatment yields higher levels of treatment uptake and engagement than AAR UC using descriptive statistics. We hypothesize that overall utilization will be higher for participants proactively enrolled in WEB + TXT compared to participants provided passive referrals to the same interventions in UC. Next, we will examine whether treatment engagement mediates the treatment-outcome relationship. To assess whether treatment engagement is predictive of improved abstinence outcomes and whether treatment engagement mediates the treatment effect, we will perform a series of additional logistic regression analyses following the framework described by Baron and Kenny [107]. The Aim 2 analytic plan is depicted in Fig. 2.

Here, $\mathrm{X}$ is the exogenous variable (treatment), $\mathrm{Z}$ is the hypothesized mediator (treatment engagement), $\mathrm{Y}$ is the outcome (abstinence at month 12), a is the regression path between treatment and the mediator, $b$ is the regression path between the mediator and the outcome, and c' is the regression path between treatment and the outcome controlling for the effect of the mediator.

To test mediation hypotheses, we will use the approach described by MacKinnon and Dwyer for binary mediator and outcome variables [108]. We will establish mediation as follows: (1) logistic regression will assess whether the effect of treatment assignment on abstinence is statistically significant; (2) logistic regression will assess whether the effect of treatment assignment on treatment use is statistically significant; (3) multivariable logistic regression will determine whether treatment use is statistically associated with abstinence after adjustment for treatment assignment; and, (4) we will assess whether the effect of treatment assignment on outcome is significantly attenuated in magnitude relative to step 1.

We will compare total and incremental costs to implement each intervention from a payer perspective. Implementation costs of the two interventions will be estimated using average market values for salaries of the additional staff needed to perform the TTS counseling and market costs of the digital intervention. If a treatment effect is observed, we will assess cost per quit and

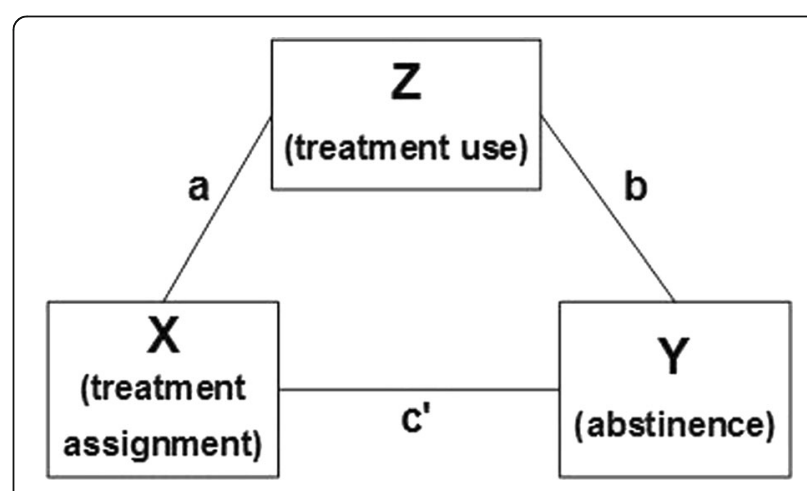

Fig. 2 Mediational model 
incremental cost per quit. Cost-effectiveness of the two proposed study interventions compared to usual care will be estimated by employing a Markov Model [109, 110]. Hypothetical cohorts representing the U.S. population will be simulated through long-term screening. Disease progression will be modeled with transition probabilities of developing lung cancer. Model parameters not directly measured in the proposed trial will be supplemented with data from the literature, including the publicly available Lung Cancer Policy Model of the Cancer Intervention and Surveillance Modeling Network [111-113]. Model complexity specific to our modeling environment will be determined by available data for the model parameters. A simplistic design not incorporating smoking status (current vs smoker) will be used as the model basis. Smoking status will be incorporated if identified data prove to make this feasible. If not feasible, the simplistic model design will serve as a crucial starting point for further analyses in the future. Sensitivity analyses will be conducted to determine the impact of changes in key model inputs.

\section{Aim 3 (Reach and Adoption) analyses}

Reach will be evaluated as the representativeness of the enrolled sample relative to all smokers that are assessed for LCS eligibility. We will compare the enrolled sample to those that are assessed for LCS eligibility on available demographic, smoking history, and medical history variables using Chi-square tests and T-tests.

Adoption will be assessed through a mixed mode quantitative/qualitative approach. We will conduct an enterprise-wide survey of LCS program directors and other key informants (e.g. nursing staff, office managers) in all LCS facilities within Mayo Clinic. The survey will gather detailed information about the barriers that would impede adoption of the digital and combined digital/clinical treatments evaluated in this study, as well as the organizational processes or resources that would facilitate their adoption and implementation. We will also conduct structured interviews with members of the Mayo Clinic Lung Screening Program to gather in-depth information about the feasibility of these treatment approaches and site-specific recommendations about their implementation.

\section{Protocol amendments}

Changes to the research protocol will be reviewed by the Institutional Review Board (IRB). Amendments will be made to the trial registry as necessary.

\section{Confidentiality}

The data management will be governed by standard procedures with regard to data security and access. All analyses are logged with respect to IRB authorization, accounting information, principal and secondary investigators, statistician, and data analyst involved in the analysis.

BecomeAnEX has stringent privacy and security policies in place which include a comprehensive privacy policy, strong encryption used in the transmission of all health-related information, electronic access controls on all personally identifiable information, and industry standard network protection. All information collected via the Internet will be kept secure in transit using the Secure Socket Layer (SSL) protocol. Both the BecomeAnEX WEB and TXT systems are cloud hosted platforms and have security audits performed annually. All communications between users and BecomeAnEX.org, and between BecomeAnEX.org and the TXT system, are sent securely via https. Both systems maintain offline backups stored in geographically separate areas for disaster recovery. All data transferred to and from the study sites will be de-identified and uploaded using a secured transfer protocol. All data will be stored in a database subject to both physical and electronic protection. The BecomeAnEX Privacy Policy is available at http://www.becomeanex.org/privacy-policy.php and will be in effect for all participants that register on the website.

\section{Dissemination}

Trial results will be posted on ClinicalTrials.gov, published in peer-reviewed journals, and presented at national conferences.

\section{Discussion}

Several key practical and operational issues warrant additional discussion. Here, we summarize key aspects of our implementation approach and the rationale underlying our decisions.

\section{Why is pharmacotherapy not provided?}

This study is designed to examine the effectiveness of interventions as delivered in LCS programs; pharmacotherapy is not typically delivered in this setting. We have aimed to strike a balance between maximizing generalizability while retaining critical elements of internal validity. All patients will be provided with a booklet that provides a rationale for medication use and describes first-line medications for smoking cessation. The WEB and TXT interventions also include information about and support for use of medication. We are interested in the extent to which each intervention arm promotes medication use, which is a dependent variable that will be examined in Aim 2.

\section{Why not compare proactive enrollment in a digital intervention to proactive enrollment in a telephone quit line?}

Although quit lines are the typical referral resource, proactive enrollment to them is not yet standard of care. 
Our decision is also rooted in an inability to track uptake and engagement of quit line resources once referred. This study will be recruiting from LCS programs in four different states that may provide different quit line services at any given time. Coordinating data exchange with quit lines is beyond the scope of this funding mechanism. A digital intervention may be delivered with complete fidelity and monitoring, regardless of patient location.

\section{Given high quit rates for TTS counseling, why not evaluate the additional impact of a digital intervention added to TTS counseling?}

Our decision was based on costs and the ease of implementation with LCS programs. TTS counseling does yield higher quit rates but at significantly higher cost $[34,35]$ and with additional considerations about how it would be incorporated into LCS workflow. Proactive enrollment in digital cessation treatments can be automated via an EHR and implemented at significantly lower cost, making it potentially more appealing for the majority of LCS settings. Aim 3 analyses will specifically address these important questions about adoption.

This study employs innovative technologies and integrated tobacco treatment modalities to promote sustainable behavior change among smokers undergoing LCS. There are few examples of fully integrated clinical and digital interventions where uptake and ongoing engagement in technology-based interventions are explicit targets of counseling. The approach in this study has the potential to be a model for not only LCS programs, but for any medical practice that treats illnesses caused by tobacco dependence. In addition, our study builds on substantial evidence for the effectiveness of proactive enrollment in cessation treatment and leverages the availability of an EHR to automate enrollment and provide real-time patient data back to the provider. EHRsupported proactive enrollment in cessation treatment has not been tested within LCS settings. There are few studies that have initiated cessation treatment immediately after shared decision-making and before LCS. This approach is designed to communicate to smokers that cessation is a priority regardless of LCS results.

Results from this study will inform the key characteristics and components of an effective smoking cessation intervention delivered in the LCS setting. Our project addresses proactive vs passive enrollment in treatment as a key element of program implementation, which has important implications for treatment uptake and engagement. The study involves proven digital (WEB and TXT) and clinical (TTS) intervention strategies and a novel approach to integrate them to address implementation challenges within the LCS setting. Our focus on the seamless integration of clinical information and behavioral health technologies aligns with federal health IT priorities in improving population health [57] and is consistent with recent guidelines for the delivery of cessation treatment in the LCS setting [19]. Our approach will inform the integration of a clinically feasible, cost-effective, and sustainable approach to tobacco dependence treatment into healthcare systems as part of usual practice.

\section{Trial status}

Study recruitment was underway at the time of manuscript submission. The trial began recruiting in August 2017.

\section{Additional file}

Additional file 1: SPIRIT Checklist. (DOC $121 \mathrm{~kb})$

\section{Abbreviations}

AAC: Ask-Advise-Connect; AAR: Ask-Advise-Refer; ACR: American College of Radiology; BOSS: Big One Stop Shop; CMS: Centers for Medicare and Medicaid Services; CO: Carbon monoxide; EHR: Electronic health record; IQR: Interquartile range; IRB: Institutional Review Board; ITT: Intent to treat; LCS: Lung cancer screening; LDCT: Low dose computed tomography; Lung-RADS: Lung imaging reporting and data system; MNAR: Missing not at random; NDC: Nicotine Dependence Center; NRT: Nicotine Replacement Therapy; PROC: Procedure; REAIM: Reach Effectiveness Adoption Implementation Maintenance; SSL: Secure socket layer; TTS: Tobacco treatment specialist; TXT: Text messaging; UC: Usual care; URL: Uniform resource locator

\section{Acknowledgements}

Not applicable.

\section{Funding}

Research reported in this publication was supported by the National Cancer Institute of the National Institutes of Health under Award Number R01CA207048. The content is solely the responsibility of the authors and does not necessarily represent the official views of the National Institutes of Health.

\section{Availability of data and materials}

The datasets generated and/or analyzed during the trial will be available from the Principal Investigator on reasonable request.

\section{Authors' contributions}

JTH and DTM are Principal Investigators of the trial. ALG drafted the manuscript. ALG, MVB, MAJ, and JTH contributed to the design of the study. SC is responsible for overall project management. ITC is responsible for development of measurement instruments and regulatory approvals and oversight. DRS developed the statistical analysis plan. JPM and BJB developed plans for evaluating cost effectiveness of the interventions. DFR oversees clinical operations and recruitment efforts across study sites. MJB and DBS are responsible for technical implementation of the BOSS system for this study and its integration with the WEB + TXT interventions. All authors provided critical revisions of the manuscript and approved the final version.

Ethics approval and consent to participate

All study participants will provide informed consent. Ethics approval was obtained from Mayo Clinic Institutional Review Board (16-004891).

Consent for publication

Not applicable. 


\section{Competing interests}

ALG, MAJ, SC, and MJB are employed by Truth Initiative, the organization that runs BecomeAnEX.

\section{Publisher's Note}

Springer Nature remains neutral with regard to jurisdictional claims in published maps and institutional affiliations.

\begin{abstract}
Author details
${ }^{1}$ Schroeder Institute for Tobacco Research and Policy Studies at Truth Initiative, 900 G Street NW, 4th Floor, Washington, DC 20001, USA. ${ }^{2}$ Department of Oncology, Georgetown University Medical Center/Cancer Prevention and Control Program, Lombardi Comprehensive Cancer Center, Washington, DC, USA. ${ }^{3}$ Mayo Clinic Nicotine Dependence Center, Mayo Clinic, Rochester, MN, USA. ${ }^{4}$ Division of Primary Care Internal Medicine, Mayo Clinic, Rochester, MN, USA. ${ }^{5}$ Division of Biomedical Statistics and Informatics, Department of Health Sciences Research, Mayo Clinic, Rochester, MN, USA. ${ }^{6}$ Kern Center for the Science of Health Care Delivery, Mayo Clinic, Rochester, MN, USA. ${ }^{7}$ Division of Health Care Policy and Research, Mayo Clinic, Rochester, MN, USA. ${ }^{8}$ Division of Research and Education Systems Support, Mayo Clinic, Rochester, MN, USA. 'Division of Pulmonary and Critical Care Medicine, Mayo Clinic, Rochester, MN, USA. ${ }^{10}$ Division of General Internal Medicine, Department of Medicine, Mayo Clinic, Rochester, MN, USA.
\end{abstract}

Received: 12 July 2017 Accepted: 1 November 2017 Published online: 28 November 2017

\section{References}

1. Ma J, Ward EM, Smith R, Jemal A. Annual number of lung cancer deaths potentially avertable by screening in the United States. Cancer. 2013;119(7):1381-5.

2. National Lung Screening Trial Research Team, Aberle DR, Adams AM, Berg CD, Black WC, Clapp JD, et al. Reduced lung-cancer mortality with low-dose computed tomographic screening. N Engl J Med. 2011;365(5): 395-409.

3. National Center for Chronic Disease Prevention and Health Promotion Office on Smoking and Health. The health consequences of smoking - 50 years of progress: a report of the Surgeon General. Atlanta: Centers for Disease Control and Prevention; 2014

4. Taylor KL, Cox LS, Zincke N, Mehta L, McGuire C, Gelmann E. Lung cancer screening as a teachable moment for smoking cessation. Lung Cancer. 2007;56(1):125-34.

5. Ostroff JS, Buckshee N, Mancuso CA, Yankelevitz DF, Henschke CI. Smoking cessation following $C T$ screening for early detection of lung cancer. Prev Med. 2001;33(6):613-21.

6. Townsend CO, Clark MM, Jett JR, Patten CA, Schroeder DR, Nirelli LM, et al. Relation between smoking cessation and receiving results from three annual spiral chest computed tomography scans for lung carcinoma screening. Cancer. 2005;103(10):2154-62.

7. Schnoll RA, Bradley P, Miller SM, Unger M, Babb J, Cornfeld M. Psychological issues related to the use of spiral CT for lung cancer early detection. Lung Cancer. 2003;39(3):315-25.

8. Slatore CG, Baumann C, Pappas M, Humphrey LL. Smoking behaviors among patients receiving computed tomography for lung cancer screening. Systematic review in support of the U.S. preventive services task force. Ann Am Thorac Soc. 2014;11(4):619-27.

9. Goffin JR, Flanagan WM, Miller AB, Fitzgerald NR, Memon S, Wolfson MC, et al. Cost-effectiveness of lung cancer screening in Canada. JAMA Oncol. 2015;1:807-13.

10. Villanti AC, Jiang $Y$, Abrams DB, Pyenson BS. A cost-utility analysis of lung cancer screening and the additional benefits of incorporating smoking cessation interventions. PLoS One. 2013;8(8):e71379.

11. Midthun DE, Jett JR. Screening for lung cancer: the US studies. J Surg Oncol. 2013;108(5):275-9.

12. Midthun DE. Early diagnosis of lung cancer. F1000Prime Rep. 2013;5:12.

13. Jensen TS, Chin J, Ashby L, Hermansen J, Hutter JD. Final national coverage determination on screening for lung cancer with low dose computed tomography (LDCT). Baltimore: Centers for Medicare and Medicaid Services; 2015. https://www.cms.gov/medicare-coverage-database/details/ncadecision-memo.aspx?NCAld=274. Accessed 20 Oct 2017.
14. Ostroff JS, Copeland A, Borderud SP, Li Y, Shelley DR, Henschke Cl. Readiness of lung cancer screening sites to deliver smoking cessation treatment: current practices, organizational priority, and perceived barriers. Nicotine Tob Res. 2016;18(5):1067-75.

15. Bentz CJ, Bayley KB, Bonin KE, Fleming L, Hollis JF, McAfee T. The feasibility of connecting physician offices to a state-level tobacco quit line. Am J Prev Med. 2006;30(1):31-7.

16. Katz DA, Muehlenbruch DR, Brown RB, Fiore MC, Baker TB, Smoking Cessation Guideline Study Group AHRQ. Effectiveness of a clinic-based strategy for implementing the AHRQ Smoking Cessation Guideline in primary care. Prev Med. 2002;35(3):293-301.

17. Solberg LI, Maciosek MV, Edwards NM, Khanchandani HS, Goodman MJ. Repeated tobacco-use screening and intervention in clinical practice: health impact and cost effectiveness. Am J Prev Med. 2006;31(1):62-71.

18. Borland R, Segan CJ. The potential of quitlines to increase smoking cessation. Drug Alcohol Rev. 2006;25(1):73-8.

19. Fucito LM, Czabafy S, Hendricks PS, Kotsen C, Richardson D, Toll BA et al. Pairing smoking-cessation services with lung cancer screening: A clinical guideline from the Association for the Treatment of Tobacco Use and Dependence and the Society for Research on Nicotine and Tobacco. Cancer. 2016;122(8):1150-9.

20. Sisterhen LL, Sheffer CE, Bursac Z, Fischer EP. Rates of enrollment in smoking cessation services following fax referrals from a children's hospital. Acad Pediatr. 2010;10(3):200-4.

21. Willett JG, Hood NE, Burns EK, Swetlick JL, Wilson SM, Lang DA, et al. Clinical faxed referrals to a tobacco quitline: reach, enrollment, and participant characteristics. Am J Prev Med. 2009;36(4):337-40.

22. Zarling KK, Burke MV, Gaines KA, Gauvin TR. Registered nurse initiation of a tobacco intervention protocol: leading quality care. J Cardiovasc Nurs. 2008;23(5):443-8.

23. Adsit RT, Fox BM, Tsiolis T, Ogland C, Simerson M, Vind LM, et al. Using the electronic health record to connect primary care patients to evidencebased telephonic tobacco quitline services: a closed-loop demonstration project. Transl Behav Med. 2014;4(3):324-32.

24. Warren GW, Marshall JR, Cummings KM, Zevon MA, Reed R, Hysert P, et al. Automated tobacco assessment and cessation support for cancer patients. Cancer. 2014;120(4):562-9.

25. Vidrine Jl, Shete S, Cao Y, Greisinger A, Harmonson P, Sharp B, et al. AskAdvise-Connect: a new approach to smoking treatment delivery in health care settings. JAMA Int Med. 2013;173(6):458-64.

26. Vidrine II, Shete S, Li Y, Cao Y, Alford MH, Galindo-Talton RM, et al. The askadvise-connect approach for smokers in a safety net healthcare system: A group-randomized trial. Am J Prev Med. 2013;45(6):737-41.

27. Pineiro B, Simmons VN, Palmer AM, Correa JB, Brandon TH. Smoking cessation interventions within the context of low-dose computed tomography lung cancer screening: A systematic review. Lung Cancer. 2016:98:91.

28. Muñoz RF, Barrera AZ, Delucchi K, Penilla C, Torres LD, Perez-Stable EJ. International Spanish/English Internet smoking cessation trial yields 20\% abstinence rates at 1 year. Nicotine Tob Res. 2009;11(9):1025-34.

29. Graham AL, Cobb NK, Papandonatos GD, Moreno JL, Kang H, Tinkelman DG et al. A randomized trial of Internet and telephone treatment for smoking cessation. Arch Intern Med. 2011;171(1):46-53.

30. Civljak M, Stead LF, Hartmann-Boyce J, Sheikh A, Car J. Internet-based interventions for smoking cessation. Cochrane Database Syst Rev. 2013;7:CD007078.

31. Shahab L, McEwen A. Online support for smoking cessation: a systematic review of the literature. Addiction. 2009;104(11):1792-804.

32. Hutton HE, Wilson LM, Apelberg BJ, Tang EA, Odelola O, Bass EB, et al. A systematic review of randomized controlled trials: Web-based interventions for smoking cessation among adolescents, college students, and adults. Nicotine Tob Res. 2011;13(4):227-38.

33. Graham AL, Carpenter KM, Cha S, Cole S, Jacobs MA, Raskob M, et al. Systematic review and meta-analysis of Internet interventions for smoking cessation among adults. Subst Abuse Rehabil. 2016;7:55-69.

34. Graham AL, Chang Y, Fang Y, Cobb NK, Tinkelman DS, Niaura RS, et al. Costeffectiveness of internet and telephone treatment for smoking cessation: an economic evaluation of The iQUITT Study. Tob Control. 2013;22(6):e11.

35. An LC, Betzner A, Schillo B, Luxenberg MG, Christenson M, Wendling $A$, et al. The comparative effectiveness of clinic, work-site, phone, and Web-based tobacco treatment programs. Nicotine Tob Res. 2010;12(10): 989-96. 
36. Bock B, Graham A, Sciamanna C, Krishnamoorthy J, Whiteley J, Carmona-Barros $\mathrm{R}$, et al. Smoking cessation treatment on the Internet: content, quality, and usability. Nicotine Tob Res. 2004;6(2):207-19.

37. Bock BC, Graham AL, Whiteley JA, Stoddard JL. A review of web-assisted tobacco interventions (WATIs). J Med Internet Res. 2008;10(5):e39.

38. Cobb NK, Graham AL, Bock BC, Papandonatos G, Abrams DB. Initial evaluation of a real-world Internet smoking cessation system. Nicotine Tob Res. 2005;7(2):207-16.

39. Ray MN, Funkhouser E, Williams JH, Sadasivam RS, Gilbert GH, Coley HL, et al. Smoking-cessation e-referrals: a national dental practice-based research network randomized controlled trial. Am J Prev Med. 2014;46(2):158-65.

40. Sadasivam RS, Hogan TP, Volkman JE, Smith BM, Coley HL, Williams JH, et al. Implementing point of care "e-referrals" in 137 clinics to increase access to a quit smoking internet system: the Quit-Primo and National Dental PBRN HI-QUIT Studies. Transl Behav Med. 2013;3(4):370-8.

41. Gordon JS, Akers L, Severson HH, Danaher BG, Boles SM. Successful participant recruitment strategies for an online smokeless tobacco cessation program. Nicotine Tob Res. 2006;8 Suppl 1:S35-41.

42. Community Preventive Services Task Force. Reducing tobacco use and secondhand smoke exposure: mobile phone-based cessation interventions. Atlanta, GA: Centers for Disease Control and Prevention; 2011

43. Fiore MC, Jaén CR, Baker TB, et al. Treating Tobacco Use and Dependence: 2008 Update. Clinical Practice Guideline. Rockville, MD: U.S. Department of Health and Human Services; 2008.

44. Abroms LC, Ahuja M, Kodl Y, Thaweethai L, Sims J, Winickoff JP, et al. Text2Quit: results from a pilot test of a personalized, interactive mobile health smoking cessation program. J Health Comm. 2012;17 Suppl 1:44-53.

45. Abroms LC, Boal AL, Simmens SJ, Mendel JA, Windsor RA. A randomized trial of Text2Quit: a text messaging program for smoking cessation. Am J Prev Med. 2014:47:242-50.

46. Brendryen $H$, Drozd F, Kraft P. A digital smoking cessation program delivered through internet and cell phone without nicotine replacement (happy ending): randomized controlled trial. J Med Internet Res. 2008;10(5):e51.

47. Brendryen $\mathrm{H}$, Kraft P. Happy ending: a randomized controlled trial of a digital multi-media smoking cessation intervention. Addiction. 2008;103(3): 478-84. discussion 485-476.

48. Whittaker R, McRobbie H, Bullen C, Rodgers A, Gu Y. Mobile phone-based interventions for smoking cessation. Cochrane Database Syst Rev. 2016:4: CD006611.

49. Guerriero C, Cairns J, Roberts I, Rodgers A, Whittaker R, Free C. The costeffectiveness of smoking cessation support delivered by mobile phone text messaging: Txt2stop. Eur J Health Econ. 2013;14(5):789-97.

50. Pew Research Center. Internet/Broadband Fact Sheet. Washington, DC: Pew Research Center; 2017. http://www.pewinternet.org/fact-sheet/internetbroadband/. Accessed 20 Oct 2017

51. Medlock S, Eslami S, Askari M, Arts DL, Sent D, de Rooij SE, et al. Health information-seeking behavior of seniors who use the Internet: a survey. J Med Internet Res. 2015;17(1):e10.

52. Pew Research Center. Mobile fact sheet. Washington, DC: Pew Research Center; 2017. http://www.pewinternet.org/fact-sheet/mobile/. Accessed 20 Oct 2017.

53. Care Innovations. Older Populations Have Adopted Technology for Health. Roseville: Care Innovations; 2013. http://resources.careinnovations.com/hs-fs/ hub/453282/file-2516634380-pdf. Accessed 20 Oct 2017.

54. Bowles KH, Hanlon A, Holland D, Potashnik SL, Topaz M. Impact of discharge planning decision support on time to readmission among older adult medical patients. Prof Case Manag. 2014;19(1):29.

55. World Health Organization. WHO Global Strategy on People-Centred and Integrated Health Services Interim Report. Geneva: World Health Organization; 2015. http://apps.who.int/iris/bitstream/10665/155002/1/ WHO_HIS_SDS_2015.6_eng.pdf?ua=1\&ua=1. Accessed 20 Oct 2017.

56. Office of the National Coordinator for Health Information Technology (ONC). Federal Health IT Strategic Plan 2015-2020. Washington, DC: U. S. Department of Health and Human Services; 2015. https://www. healthit.gov/sites/default/files/federal-healthlT-strategic-plan-2014.pdf. Accessed 20 Oct 2017.

57. Zbikowski SM, Magnusson B, Pockey JR, Tindle HA, Weaver KE. A review of smoking cessation interventions for smokers aged 50 and older. Maturitas. 2012;71(2):131-41.

58. Shields PG, Herbst RS, Arenberg D, Benowitz NL, Bierut L, Luckart JB, et al. Smoking Cessation, Version 1.2016, NCCN Clinical Practice Guidelines in Oncology. J Natl Compr Canc Netw. 2016;14(11):1430-68.
59. Hurt RD, Ebbert JO, Hays JT, McFadden DD. Treating tobacco dependence in a medical setting. CA Cancer J Clin. 2009;59(5):314-26.

60. Burke MV, Ebbert JO, McFadden D, Hays JT. Treatment outcomes from a specialist model for treating tobacco use disorder in a medical center. Medicine. 2015;94(44):e1903.

61. Hurt RD, Ebbert JO, Hays JT, McFadden DD. Preventing lung cancer by treating tobacco dependence. Clin Chest Med. 2011;32(4):645-57.

62. Kotz D, Brown J, West R. Prospective cohort study of the effectiveness of smoking cessation treatments used in the "real world". Mayo Clin Proc. 2014:89(10):1360-7.

63. Garces YI, Schroeder DR, Nirelli LM, Croghan GA, Croghan IT, Foote RL, et al. Tobacco use outcomes among patients with head and neck carcinoma treated for nicotine dependence: a matched-pair analysis. Cancer. 2004;101(1):116-24.

64. Khara M, Okoli C, Nagarajan VD, Aziz F, Hanley C. Smoking cessation outcomes of referral to a specialist hospital outpatient clinic. Am J Addict. 2015;24(6):561-70.

65. Hughes JR. Tobacco treatment specialists: a new profession. J Smoking Cessation. 2007;2:2-7.

66. Song F, Maskrey V, Blyth A, Brown TJ, Barton GR, Aveyard P, et al. Differences in longer-term smoking abstinence after treatment by specialist or nonspecialist advisors: secondary analysis of data from a relapse prevention trial. Nicotine Tob Res. 2016;18:1061-6.

67. Ferketich AK, Otterson GA, King M, Hall N, Browning KK, Wewers ME. A pilot test of a combined tobacco dependence treatment and lung cancer screening program. Lung Cancer. 2012;76(2):211-5.

68. Reid RD, Mullen KA, Slovinec D'Angelo ME, Aitken DA, Papadakis S, Haley PM, et al. Smoking cessation for hospitalized smokers: an evaluation of the "Ottawa Model". Nicotine Tob Res. 2010;12(1):11-8.

69. Zbikowski SM, Hapgood J, Smucker Barnwell S, McAfee T. Phone and webbased tobacco cessation treatment: real-world utilization patterns and outcomes for 11,000 tobacco users. J Med Internet Res. 2008;10(5):e41.

70. Glasgow RE, Vogt TM, Boles SM. Evaluating the public health impact of health promotion interventions: the RE-AIM framework. Am J Public Health. 1999;89(9):1322-7.

71. Tunis SR, Stryer DB, Clancy CM. Practical clinical trials: increasing the value of clinical research for decision making in clinical and health policy. JAMA. 2003:290(12):1624-32.

72. Moyer VA, Force USPST. Screening for lung cancer: U.S. Preventive Services Task Force recommendation statement. Ann Intern Med. 2014; 160(5):330-8.

73. Tammemagi MC, Katki HA, Hocking WG, Church TR, Caporaso N, Kvale PA, et al. Selection criteria for lung-cancer screening. N Engl J Med. 2013;368(8):728-36.

74. Mayo Foundation for Medical Education and Research. Patient Education: Medications to Help You Stop Using Tobacco. Rochester, MN: Mayo Foundation for Medical Education and Research; 2014.

75. McCausland KL, Curry LE, Mushro A, Carothers S, Xiao H, Vallone DM. Promoting a web-based smoking cessation intervention: implications for practice. Cases Public Health Commun Mark. 2011;5:3-26.

76. Richardson A, Graham AL, Cobb N, Xiao H, Mushro A, Abrams D, et al. Engagement promotes abstinence in a web-based cessation intervention: cohort study. J Med Internet Res. 2013;15(1):e14.

77. Bandura A. Self-efficacy: toward a unifying theory of behavioral change. Psychol Rev. 1977:84(2):191-215.

78. Czaja SJ, Lee CC. Older Adults and Information Technology: Opportunities and Challenges. In: Jacko JA, editor. The Human-Computer Interaction Handbook: Fundamentals, Evolving Technologies and Emerging Applications. Boca Raton: Taylor \& Francis; 2012. p. 825-40.

79. National Institute on Aging: Making Your Website Senior Friendly. 2011. https://www.nlm.nih.gov/pubs/checklist.pdf. Accessed 20 Oct 2017.

80. Arch A, Abou-Zahra S. Web accessibility and older people: meeting the needs of ageing web users. 2010. https://www.w3.org/WAl/older-users/ Overview.php. Accessed 20 Oct 2017.

81. Chisnell D, Redish J. Designing web sites for older adults: expert review of 50 web sites. 2004. https://assets.aarp.org/www.aarp.org_/articles/research/ oww/AARP-LitReview2004.pdf. Accessed 20 Oct 2017.

82. Centers for Medicare \& Medicaid Services. Decision memo for screening for lung cancer with low dose computed tomography (LDCT) (CAG-00439 N). Baltimore, MD: Centers for Medicare and Medicaid, U.S. Department of Health and Human Services. https:/www.cms.gov/medicare-coverage-database/ details/nca-decision-memo.aspx?NCAld=274. Accessed 20 Oct 2017. 
83. Kazerooni EA, Austin JH, Black WC, Dyer DS, Hazelton TR, Leung AN, et al. ACR-STR practice parameter for the performance and reporting of lung cancer screening thoracic computed tomography (CT): 2014 (Resolution 4). J Thorac Imaging. 2014:29(5):310-6.

84. Shah A, Yon A, Scogin F. Clinical research with older adults. In: McKay D, editor. Handbook of research methods in abnormal and clinical psychology. Thousand Oaks, CA: SAGE Publications, Inc; 2008. p. 427-46.

85. Hurt RD, Sachs DP, Glover ED, Offord KP, Johnston JA, Dale LC, et al. A comparison of sustained-release bupropion and placebo for smoking cessation. N Engl J Med. 1997;337(17):1195-202.

86. Jorenby DE, Hays JT, Rigotti NA, Azoulay S, Watsky EJ, Williams KE, et al. Efficacy of varenicline, an alpha4beta2 nicotinic acetylcholine receptor partial agonist, vs placebo or sustained-release bupropion for smoking cessation: a randomized controlled trial. JAMA. 2006;296(1):56-63.

87. Swensen SJ, Jett JR, Hartman TE, Midthun DE, Mandrekar SJ, Hillman SL, et al. CT screening for lung cancer: five-year prospective experience. Radiology. 2005;235(1):259-65.

88. Biener L, Abrams DB. The Contemplation Ladder: validation of a measure of readiness to consider smoking cessation. Health Psychol. 1991;10(5):360-5.

89. Herzog TA, Abrams DB, Emmons KM, Linnan LA. Predicting increases in readiness to quit smoking: A prospective analysis using the contemplation ladder. Psychol Health. 2000;15:369-81.

90. Heatherton TF, Kozlowski LT, Frecker RC, Fagerstrom KO. The Fagerstrom Test for Nicotine Dependence: a revision of the Fagerstrom Tolerance Questionnaire. Br J Addict. 1991:86(9):1119-27.

91. SRNT Subcommittee on Biochemical Verification. Biochemical verification of tobacco use and cessation. Nicotine Tob Res. 2002;4(2):149-59.

92. Hughes JR, Keely JP, Niaura RS, Ossip-Klein DJ, Richmond RL, Swan GE. Measures of abstinence in clinical trials: issues and recommendations. Nicotine Tob Res. 2003;5(1):13-25.

93. Nguyen TD, Attkisson CC, Stegner BL. Assessment of patient satisfaction: development and refinement of a service evaluation questionnaire. Eval Program Plann. 1983;6(3-4):299-313.

94. Usability.gov. System Usability Scale (SUS). https://www.usability.gov/how to-and-tools/methods/system-usability-scale.html. Accessed 20 Oct 2017.

95. Olufade AO, Shaw JW, Foster SA, Leischow SJ, Hays RD, Coons SJ. Development of the Smoking Cessation Quality of Life questionnaire. Clin Ther. 1999:21(12):2113-30.

96. Ware Jr JE, Sherbourne CD, The MOS. 36-item short-form health survey (SF-36). I. Conceptual framework and item selection. Med Care. 1992;30(6):473-83.

97. Tammemagi MC, Berg CD, Riley TL, Cunningham CR, Taylor KL. Impact of lung cancer screening results on smoking cessation. J Natl Cancer Inst. 2014;106(6):dju084.

98. Ashraf H, Tonnesen P, Holst Pedersen J, Dirksen A, Thorsen H, Dossing M. Effect of $C T$ screening on smoking habits at 1-year follow-up in the Danish Lung Cancer Screening Trial (DLCST). Thorax. 2009;64(5):388-92.

99. van der Aalst CM, van Klaveren RJ, van den Bergh KA, Willemsen MC, de Koning HJ. The impact of a lung cancer computed tomography screening result on smoking abstinence. Eur Respir J. 2011;37(6):1466-73.

100. Park ER, Gareen IF, Jain A, Ostroff JS, Duan F, Sicks JD, et al. Examining whether lung screening changes risk perceptions: National Lung Screening Trial participants at 1-year follow-up. Cancer. 2013;119(7):1306-13.

101. Cox LS, Clark MM, Jett JR, Patten CA, Schroeder DR, Nirelli LM, et al. Change in smoking status after spiral chest computed tomography scan screening. Cancer. 2003;98(11):2495-501.

102. Webb TL, Joseph J, Yardley L, Michie S. Using the internet to promote health behavior change: a systematic review and meta-analysis of the impact of theoretical basis, use of behavior change techniques, and mode of delivery on efficacy. J Med Internet Res. 2010;12(1):e4.

103. Borland R, Balmford J, Benda P. Population-level effects of automated smoking cessation help programs: a randomized controlled trial. Addiction. 2013;108(3):618-28.

104. Holm S. A simple sequentially rejective multiple test procedure. Scand J Statist. 1979;6(2):65-70

105. Carpenter J, Kenward M. Multiple imputation and its application. West Sussex, UK: John Wiley \& Sons; 2013.

106. van Buuren S. Flexible imputation of missing data. Boca Raton, FL: Taylor \& Francis Group; 2012

107. Baron RM, Kenny DA. The moderator-mediator variable distinction in social psychological research: conceptual, strategic, and statistical considerations. J Pers Social Psych. 1986;51(6):1173-82.
108. Mackinnon DP, Dwyer JH. Estimating mediated effects in prevention studies. Eval Rev. 1993;17(2):144-58.

109. Sonnenberg FA, Beck JR. Markov models in medical decision making: a practical guide. Med Decis Making. 1993;13(4):322-38.

110. Ramsey SD, Willke RJ, Glick H, Reed SD, Augustovski F, Jonsson B, et al. Cost-effectiveness analysis alongside clinical trials II-An ISPOR Good Research Practices Task Force report. Value Health. 2015;18(2):161-72.

111. McMahon PM, Kong CY, Bouzan C, Weinstein MC, Cipriano LE, Tramontano AC, et al. Cost-effectiveness of computed tomography screening for lung cancer in the United States. J Thorac Oncol. 2011;6(11):1841-8.

112. McMahon PM, Kong CY, Johnson BE, Weinstein MC, Weeks JC, Kuntz KM, et al. Estimating long-term effectiveness of lung cancer screening in the Mayo CT screening study. Radiology. 2008;248(1):278-87.

113. MCMahon PM, Kong CY, Weinstein MC, Tramontano AC, Cipriano LE, Johnson BE, et al. Adopting helical $\mathrm{CT}$ screening for lung cancer: potential health consequences during a 15-year period. Cancer. 2008;113(12):3440-9.

\section{Submit your next manuscript to BioMed Central and we will help you at every step:}

- We accept pre-submission inquiries

- Our selector tool helps you to find the most relevant journal

- We provide round the clock customer support

- Convenient online submission

- Thorough peer review

- Inclusion in PubMed and all major indexing services

- Maximum visibility for your research

Submit your manuscript at www.biomedcentral.com/submit
C Biomed Central 